\title{
Assisted reproductive technology
}

\author{
Simon M Kelly MB ChB FRANZCOG, Seang Lin Tan MBBS FRCOG FRCSC MMed (O\&G)
}

\section{SM Kelly, SL Tan. Assisted reproductive technology. J Sex Reprod Med 2002;2(4):153-159.}

Since the first successful in vitro fertilization (IVF) pregnancy 24 years ago, the progress of assisted reproduction has been astounding. IVF remains the cornerstone of fertility treatment, and there have been many significant clinical and embryological advances. The success rates of treatment continue to improve steadily, and with developments such as intracytoplasmic sperm injection there are many more couples who can benefit from assisted reproductive technologies. Cryopreservation techniques are being improved continually, and with its application to oocyte and ovarian tissue freezing, female fertility preservation is fast becoming a reality. Newer developments involving in vitro maturation of immature oocytes, preimplantation diagnosis and aneuploidy screening are discussed, as well as the risks and complications associated with assisted reproductive technologies. The rapid growth of this technology has also been paralleled by a number of ethical and social dilemmas that society has, in many circumstances, struggled to reach a consensus on.

Key Words: Cryopreservation; Intracytoplasmic sperm injection; In vitro fertilization; Ovarian hyperstimulation syndrome

\section{Techniques de reproduction assistée}

RÉSUMÉ: Depuis la première grossesse obtenue par fécondation in vitro (FIV) il y a 24 ans, les progrès de la reproduction assistée sont étonnants. La FIV demeure la pierre angulaire du traitement de l'infertilité, et des progrès considérables ont été réalisés sur les plans embryologique et clinique. Le taux de succès du traitement ne cesse d'augmenter. De plus, la mise au point de procédés comme l'injection intracytoplasmique de spermatozoïdes permet à de plus en plus de couples de profiter des techniques de reproduction assistée (TRA). En outre, les méthodes de cryoconservation s'améliorent toujours et, grâce à leur application à la congélation d'ovocytes et de tissu ovarien, la préservation de la fertilité féminine devient rapidement réalité. Il sera également question d'autres progrès, dont la maturation in vitro d'ovocytes immatures, le diagnostic de préimplantation, le dépistage de l'aneuploïdie, de même que les risques et les complications liés aux TRA. L'évolution rapide de ces techniques a engendré certains dilemmes éthiques et sociaux sur lesquels la société tente, dans bien des cas, de faire consensus.

McGill Reproductive Centre, Department of Obstetrics and Gynecology, McGill University, Montreal, Quebec

Correspondence: Dr Simon M Kelly, McGill Reproductive Centre, Royal Victoria Hospital, 687 Pine Avenue West, Montreal, Quebec

H3A 1A1. Telephone 514-843-1231 ext 36861, fax 514-843-1496, e-mail simon.kelly@muhc.mcgill.ca 
A ssisted reproductive technology (ART) is a global term used to describe the myriad of advanced infertility treatment options available to help couples with infertility achieve a pregnancy. From a practical perspective, the techniques of in vitro fertilization (IVF) and intracytoplasmic sperm injection (ICSI) are the cornerstones of modern ART.

Louise Brown's birth in July 1978, as the first successful pregnancy resulting from IVF, was the culmination of many years of work by the fathers of ART, Patrick Steptoe and Robert Edwards (1).

These early developments provided the launching pad for an explosion in the use of IVF and other assisted reproductive techniques (2-4). It is estimated that about one million babies have been born as a result of IVF treatment worldwide.

\section{IVF}

Although IVF was developed initially as a treatment for tubal factor infertility, it has many more indications. With new developments, particularly in relation to male factor infertility, there are many more couples nowadays for whom treatment provides an opportunity to have their own child.

As mentioned previously, IVF is the prime component of assisted reproduction. There are a variety of IVF protocols that may be instituted, depending on patient characteristics or clinician preference. A standard approach to IVF treatment is outlined below.

\section{Pretreatment assessment}

It is important that couples are counselled appropriately regarding the likely success rates of treatment, as well as associated risks, including multiple pregnancy and ovarian hyperstimulation syndrome (OHSS). From a clinical perspective, the likely response to ovarian stimulation is assessed by the evaluation of early follicular phase levels of follicle-stimulating hormone (FSH) and estradiol, a baseline ultrasound scan to assess the presence or absence of polycystic ovaries (PCO) (5), and measuring the antral follicle count and the intraovarian stromal blood flow velocity $(6,7)$.

\section{Pituitary downregulation}

Most current IVF treatments use a standard long protocol gonadotropin-releasing hormone $(\mathrm{GnRH})$ agonist prior to ovarian stimulation to achieve pituitary suppression (8). This normally requires seven to 21 days of GnRH-a administration. This has the advantage of reducing luteinizing hormone (LH) levels during ovarian stimulation, which may, if present in high levels, be detrimental to oocyte quality and subsequent fertilization and implantation rates (9). In addition, continuing GnRH agonists during stimulation will minimize the risk of premature LH surge and ovulation (10).

\section{Controlled ovarian stimulation}

Once pituitary suppression is accomplished, ovarian stimulation is achieved by exogenous gonadotropin (FSH) stim- ulation. There are a variety of preparations available that are broadly divided into either urinary derived products or recombinant products. Urinary products generally include a variable amount of LH activity, whereas recombinant products are pure FSH. There is ongoing debate regarding the possible deleterious effects and amount of LH activity required for optimal ovarian stimulation (11). Follicular growth can be assessed accurately by transvaginal ultrasound, with the addition of estradiol evaluation in selected situations (poor or excessive response).

Most protocols continue stimulation until there are at least three follicles with mean diameters of $18 \mathrm{~mm}$ or greater.

\section{Ovulation trigger with human chorionic gonadotrophin} Once the leading follicles reach the appropriate dimensions, human chorionic gonadotrophin (hCG) is administered as a surrogate for the normal LH surge to induce final oocyte maturation within the follicles.

\section{Oocyte collection}

Ultrasound directed oocyte collection is almost universal. It means that IVF can largely be done as an outpatient procedure, without the need for anesthesia (12).

With a needle guide attached to a vaginal transducer, a double lumen needle is inserted through the posterolateral vaginal fornix into a selected follicle in the adjacent ovary. A vacuum pump is used to aspirate follicular fluid into a collecting tube. The embryologist examines the aspirate to detect the presence of the oocyte. Each follicle should be emptied completely to ensure a maximum oocyte recovery rate. Flushing of the follicle should be performed sparingly, if at all (13).

\section{Insemination and embryo culture}

Most oocytes are surrounded by a layer of cumulus cells (oocyte cumulus complex). Insemination is performed within $6 \mathrm{~h}$ following the collection of the oocytes. For routine IVF, a prepared sperm sample is added to each oocyte cumulus complex. For ICSI, the cumulus cells are stripped away and only metaphase II oocytes are injected.

A check is performed for fertilization 18 to $20 \mathrm{~h}$ following insemination. The presence of two pronuclei confirms normal fertilization. Culture is continued for 24 to $36 \mathrm{~h}$ before a further assessment for embryo cleavage is performed. At this stage, the embryos are normally between the two to eight cell (blastomere) stage. Embryo quality is assessed depending on blastomere symmetry and the degree of cytoplasm fragmentation before transfer.

\section{Embryo transfer}

Classically, embryos are transferred on either day 2 or day 3 following oocyte collection. The number of embryos transferred depends on a variety of factors, including the age of the female patient, previous number of IVF treatments, embryo quality and number, the wishes or concerns of the couple in relation to multiple pregnancy and any legislation relating to 
the maximum number of embryos that may be transferred.

More recently, the use of a sequential culture media has enhanced the rate of formation of blastocysts in the laboratory, and the embryo transfer may be delayed to day 5 . Blastocyst culture is an attempt to select embryos with higher implantation potential and to minimize multiple pregnancy.

\section{Luteal support}

The secretion of progesterone from the corpus luteum is essential for the support of early pregnancy. There are two main factors that may imply a deficient luteal phase in the context of an IVF cycle. The very nature of follicle aspiration means that many granulosa cells may have been removed or disrupted. In addition, there is evidence that the use of GnRH agonist in IVF treatment has a negative impact on corpora lutea function.

For these reasons, the luteal phase is supported by the administration of progesterone, either in the form of vaginal pessaries or as daily intramuscular injections. As an alternative, some units will use low dose hCG injections instead of progesterone to support luteal function. Both are equally efficacious but hCG may increase the risk of OHSS.

The woman normally has a pregnancy test performed 14 days after embryo transfer, and, if pregnant, continues luteal support until eight to 12 weeks' gestation.

\section{ICSI}

Soon after IVF became an established treatment for a variety of fertility problems, it also became apparent that couples with a severe male factor problem were often not helped by traditional IVF. In a situation where the sperm count or motility was very low, or there were high numbers of abnormal sperm, IVF often led to failed fertilization. A variety of sperm injection techniques were tried, but ultimately ICSI of sperm proved to be the most successful. It is now practised routinely by many units and is indicated in a number of situations, including severe oligospermia, marked asthenospermia (low motility), elevated numbers of morphologically abnormal sperm (less than 15\% normal morphology), previous fertilization failure with conventional IVF or when epididymal or testicular sperm is used.

In men who produce no sperm (or no viable sperm) in the ejaculate, there are a variety of surgical techniques to retrieve sperm from either the epididymis or from the testicle itself. Surgical sperm retrieval techniques are discussed elsewhere in this issue of the journal.

\section{CRYOPRESERVATION IN ART}

The development of IVF programs using ovarian stimulation often means that following the transfer of a selected number of fresh embryos, a number of embryos may remain. It was not long before the science of cryobiology was applied and remaining embryos were frozen for future use. Most embryos are frozen using a slow cooling process with the addition of a cryoprotectant to avoid intracellular ice formation. These embryos may be thawed and replaced at a later date as an alternative to further stimulated cycles of IVF. Embryos may be frozen at pronucleate, cleavage or blastocyst stages of development. When patients have a large number of embryos, it is advisable to consider freezing a portion at the pronucleate phase, because they are less likely to suffer damage to the mitotic spindle than are cleaved embryos. They subsequently yield a higher pregnancy rate following thawing and transfer (14).

Around $70 \%$ to $80 \%$ of embryos survive thawing, depending on their initial quality. Once thawed, the embryos are placed in culture, and, if frozen at pronucleate phase, are allowed to cleave before transfer. Transfer of embryos may be done in a natural menstrual cycle, or, if the woman has an irregular cycle, pituitary downregulation with GnRH agonists following hormone replacement therapy may be used.

More recently, cryobiology has been applied to the freezing of oocytes and ovarian tissue. The survival of the oocytes following thawing is extremely low compared with that of embryo cryopreservation. There also appears to be an increased risk of aneuploidy when metaphase II oocytes are cooled or warmed. The freezing of immature oocytes has been attempted, but again, the results have been poor. Nevertheless, it is an attractive option for fertility preservation in women undergoing chemotherapy or radiotherapy for various malignancies.

Cryopreservation of ovarian tissue is also an attractive option for women wanting to preserve fertility. The ovarian cortex contains many primordial follicles, but despite the existence of viable follicles following thawing, the currently available culture media are inadequate to maintain in vitro maturation (IVM).

Alternatively, it is possible that harvested ovarian tissue may be autografted back to the donor to restore fertility. There has been some success reported in the sheep model (15) and at least one report of partial restoration of ovarian function in a woman who underwent autologous grafting (16). There are concerns, however, in transplanting ovarian tissue back to cancer patients, particularly when there may be an etiological link to the ovary (ie, breast cancer).

Vitrification is a cryopreservation technique that is an alternative to the traditional slow freezing method. Essentially it involves a process of solidification of a solution (ie, within the cytoplasm of a cell) at a low temperature without ice crystal formation. It uses a higher concentration of cryoprotectant that is potentially more toxic to the cell, and it is then cooled at a higher rate. The technique is much simpler and is less expensive than the traditional slow cooling method. It has been used successfully with both oocytes and embryos and may be the method of choice, particularly for oocyte freezing (17).

\section{OOCYTE DONATION}

The first reported pregnancy following the donation of an oocyte fertilized in vitro was in 1983 (18). Excellent pregnancy and live birth rates can be expected with the appropriate selection of donors. Initially, it was used as a 
treatment for women with premature ovarian failure, but nowadays it has wider applications, including infertility treatment in older patients who produce poor quality oocytes following conventional IVF, or in women who are carriers of specific genetic conditions that are transmitted by the oocyte. It is also often advocated in women with recurrent IVF failures. In theory, there is no age barrier to successful oocyte donation.

Unfortunately, the demand for donor oocytes outweighs the supply of donor oocytes, so that many units have very long waiting lists of patients requiring donor oocytes. Donors may be altruistic volunteers or known to the recipient (often a friend or relative). Anonymity, even with a known donor, can be achieved using a crossover pairing of two sets of donors and recipients.

Egg sharing is a program that is increasingly being offered in some units. Patients undergoing IVF may volunteer to donate any excess oocytes to an anonymous recipient. The costs of treatment may be shared between the couples.

\section{NEW DEVELOPMENTS IN ART}

Assisted reproduction is a rapidly developing area of medicine and we review a number of the more recent advances.

\section{IVM}

The collection of immature oocytes from unstimulated ovaries with subsequent IVM and fertilization is receiving increasing interest as an alternative to traditional IVF.

Initially, pregnancy rates following IVM were low. More recently, pregnancy rates in excess of $25 \%$ have been reported particularly in women with PCO (19-21).

The concept of IVM is very attractive for a number of reasons. The costs of treatment are reduced because patients do not require medications for ovarian stimulation. The avoidance of ovarian stimulation also means that side effects due to the use of gonadotropins are also avoided. Most importantly, the risk of OHSS is avoided and this is especially important to those patients with $\mathrm{PCO}$ who have an increased risk of OHSS with gonadotropin stimulation. In addition, there has been concern expressed over the possible long term risk of ovarian cancer following repeated cycles of ovarian stimulation (22), which would not be a concern with IVM treatment.

Patients undergoing an IVM cycle have a baseline ultrasound scan performed between days 2 and 4 of their cycle. At this point, the total number of antral follicles of greater than $2 \mathrm{~mm}$ to $4 \mathrm{~mm}$ is assessed. It has been demonstrated that the success of IVM depends on the number of immature oocytes obtained, which in turn can be predicted on the basis of the antral follicle count (23). Pregnancy rates comparable with conventional IVF may be obtained when 10 or more immature oocytes are collected. Oocytes can be retrieved from about $50 \%$ of visible follicles. This implies that patients who have $\mathrm{PCO}$ (greater than 10 follicles per ovary) visible on ultrasound could be offered IVM treatment as an alternative to IVF (24).
The patient is normally scanned again on day 8 , and oocyte retrieval is planned for between days 10 and 14 . Thirty-six hours before oocyte collection, a subcutaneous injection of hCG is given. This has been shown to improve the rate of maturation of the immature oocytes and the final number of mature oocytes obtained (25).

The cycle is normally cancelled if a dominant follicle (greater than $10 \mathrm{~mm}$ ) is present on day 8 because initial reports have suggested a reduced pregnancy rate. The collection of immature oocytes is performed under ultrasound guidance with a reduced aspiration pressure. Although similar to IVF, it is a more technically challenging procedure.

Following collection, the immature oocytes are cultured for $24 \mathrm{~h}$ before fertilization (by ICSI) of the metaphase II oocytes. Some oocytes require up to $48 \mathrm{~h}$ to mature. Maturation rates of approximately $80 \%$ have been reported. Embryo transfer is performed two to three days later.

The endometrium is also primed with estradiol valerate from the day of collection and progesterone luteal support is given from the day of ICSI.

At present, IVM is most applicable to patients with PCO. With continued improvements, it may also become an option for women with normal ovaries and those who have repeatedly poor quality embryos following conventional IVF. In addition, it may be a viable alternative for egg donors, particularly those with PCO.

\section{Preimplantation diagnosis}

Although not strictly a new technique, preimplantation genetic diagnosis (PGD) is gaining more widespread utilization. The first birth of a child following PGD was over a decade ago (26), and the births of more than 500 babies have been reported following PGD.

The technique essentially involves removing one or two blastomeres (which are representative of the embryo) from an eight cell embryo three days after fertilization. There are two techniques used to analyze the genetic status of the blastomere. First, polymerase chain reaction is used to amplify a particular genetic sequence, and then, with test samples and DNA gel electrophoresis, a mismatch will identify a specific mutation. It can be used for carriers of single gene defects involving autosomes and for sex chromosome linked disorders.

Second, fluorescent in situ hybridization is used to determine the ploidy of the blastomere by using probes to bind specific chromosomes. Up to eight chromosomes may be tested using this process (usually X, Y, 13, 15, 16, 18, 21 and 22). The technique has been shown to reduce the rate of trisomic offspring, increase implantation rates and reduce the rate of spontaneous abortion. PGD can be used for aneuploidy screening, sex selection for X-linked disorders and identification of unbalanced translocations from parental carriers (27). Once the results of analysis are known, the unaffected embryos are reimplanted. Most embryos survive biopsy; however, pregnancy rates are lower than those achieved with conventional IVF. The remaining embryos are usually retested as a method of quality control. 
Cryopreservation of embryos following biopsy has not proved to be very successful.

PGD has gained a lot of interest, particularly in relation to aneuploidy screening in women over the age of 37 years undergoing IVF (28). It is well recognized that the proportion of embryos that have chromosomal abnormalities rises with increasing female age. The benefits in terms of improved pregnancy rates and reduced rates of miscarriage are significant in this age group as long as the cohort of embryos is greater than would normally be transferred for a given age. PGD can be viewed as an adjunct to antenatal diagnosis, although the long term safety is unknown.

One of the limitations of current PGD practice is that two-thirds of the human chromosome complement are untested. Comparative genomic hybridization is a variation of PGD that allows the visualization of all 46 chromosomes following amplification of the whole test genome. It is then labelled with one colour and compared with a normal reference sample. Specially developed image processing software can delineate structural differences between the test sample and the control. Although the technique is not yet at a stage to be of practical use, it may well be the way forward for embryo selection beforetransfer by improving the detection of aneuploidic embryos (29).

\section{New drug regimens}

Two of the disadvantages of most current IVF drug regimens are the duration of administration of the medications, and that they require subcutaneous or intramuscular injections. More recently, a new variety of medication has become available as an alternative to the traditional GnRH agonist long protocol regimen. GnRH antagonists were developed in the early 1970s, but they were not clinically suitable because they were associated with a significant histamine release at the site of injection. A new generation of $\mathrm{GnRH}$ antagonists is available. They have an advantage over GnRH agonists in that they cause immediate and transient pituitary suppression. In addition, when used in conjunction with gonadotropin stimulation, a lower dose of stimulation is often required. The antagonist may be administered as a single or multiple dose protocol. It is commenced normally around day 6 of gonadotropin stimulation or when the leading follicles are 12 $\mathrm{mm}$ to $14 \mathrm{~mm}$ in diameter. It is continued until the day of hCG administration and appears to be as effective as the GnRH agonist protocols in preventing premature LH surges.

The new drug regimen allows for a more flexible and patient friendly IVF protocol; however, most studies suggest that, at present, the implantation and pregnancy rate with $\mathrm{GnRH}$ antagonists are lower than when the long protocol $\mathrm{GnRH}$ agonists are used. Research is also underway to develop long-acting slow-release gonadotrophin preparations and ultimately an oral form.

\section{RESULTS OF ASSISTED REPRODUCTION}

There are many variables that affect the chances of a successful outcome for a couple. Paramount is the age of the female partner, and success rates of IVF and all fertility treatments reflect this. Although age is a guide to success rates, other factors such as ovarian reserve, length of infertility, previous treatment history, cause of infertility, the presence of a normal uterine cavity and the number of embryos transferred will have a bearing in individual cases.

Many IVF units now quote clinical pregnancy rates in the order of $35 \%$ to $40 \%$ per cycle in women up to their mid to late 30 s. Women over the age of 40 years may expect success rates of less than $20 \%$. Thereafter, there is a significant reduction, with extremely few women over the age of 44 to 45 years of age conceiving with IVF.

As can be deduced from these figures, the majority of couples will not conceive following a single cycle of treatment. However, a recent study reported that for women younger than 30 years of age, the cumulative pregnancy and live birth rates after three cycles of treatment were $75.5 \%$ and $71.8 \%$, respectively. For all women treated up to the age of 45 years, the cumulative pregnancy and live birth rates after three cycles were $54.2 \%$ and $48.2 \%$, respectively (30). With new developments in aneuploidy screening of embryos before replacement, there is potential to significantly improve success rates in the future.

\section{COMPLICATIONS AND LIMITATIONS OF ART}

There are a number of potential complications that may arise as a result of ART. These complications may occur during the treatment cycle itself or as a result of the pregnancy achieved by treatment.

The development of ovarian cysts during IVF treatment is not uncommon and may occur as a result of pituitary suppression from $\mathrm{GnRH}$ agonist use or from previous gonadotrophin therapy. The cysts are generally not a significant health risk and are dealt with relatively easily. When they occur following GnRH agonist therapy, continuing treatment for a further week may lead to resolution or the cysts may be aspirated, particularly if they are large.

The procedure of oocyte collection and embryo transfer can also lead to complications of infection or hemorrhage, although this is not common. Patients with ovarian endometriosis appear to have a higher risk of infection and prophylactic antibiotics should be administered.

The main concern of most IVF practitioners is the development of OHSS. This is a potentially life threatening iatrogenic condition resulting from excessive ovarian stimulation. It is characterized in its most severe form by enlarged ovaries, ascites, pleural effusions and subsequent dehydration and hemoconcentration.

Thromboembolism, which is potentially fatal, may subsequently develop (31). Severe OHSS may occur in up to $1 \%$ of patients undergoing IVF and a further $10 \%$ may have mild to moderate OHSS. It may develop early following oocyte retrieval, or arise a week or so after embryo transfer, when it is often associated with pregnancy. Patients with polycystic ovarian syndrome, lean body mass or a history of OHSS are most at risk. During gonadotrophin therapy, the development of multiple follicles (more than 20), especial- 
ly medium sized follicles $12 \mathrm{~mm}$ to $14 \mathrm{~mm}$ in diameter, often with a concurrent excessively elevated serum estradiol level (greater than $10,000 \mathrm{pmol} / \mathrm{L}$ ) should alert the clinician to the risk of OHSS. Strategies aimed at the prevention of OHSS may include reducing the hCG trigger dose (5000 U), coasting the cycle by withholding stimulation until the estradiol has fallen, and proceeding with collection but freezing all embryos or cancellation of the cycle. Once OHSS is established, the aims of treatment are essentially supportive, with rehydration and replacement of albumin, ascitic drainage as required, and anticoagulation.

Successful ART is often characterized by a higher chance of multiple pregnancy and the incumbent medical, social and economical consequences (32). At present, the only effective means of reducing the multiple pregnancy rate is to limit the number of embryos transferred. There is concern that this will reduce overall pregnancy rates, although some evidence suggests that electively reimplanting only two embryos in young women who produce many good quality embryos reduces triplet pregnancy rates without affecting overall success rates. In our centre, we have adopted this policy for the past six years, and high pregnancy rates have been maintained with a triplet pregnancy rate of less than $4 \%$. In comparison, a recent analysis of data from all centres in the United States shows an overall triplet pregnancy rate in excess of $8 \%$ (33). It is hoped that with improved aneuploidy screening techniques, a reduction in the number of embryos being reimplanted can be achieved with either similar or better pregnancy rates.

Pregnancy complications following ART have been well studied. The rates of preterm delivery and low birth weight appear to be more common, even in singleton pregnancies, as a result of ART (34). However, the majority of problems are a consequence of the increased multiple pregnancy rates associated with treatment, and also most probably the infertility status of these patients (35).

The overall risk of congenital malformations following ART is not significantly different from that of the general population when adjusted for age and multiple pregnancy. However, the incidence of sex chromosomal abnormalities may be increased from $0.2 \%$ to $0.7 \%$ following ICSI. This is most likely a result of subtle chromosomal abnormalities in the men with severe sperm problems for which ICSI is typically used (36).

A few years ago, it was suggested that repeated cycles of stimulation (more than 12) without pregnancy may be associated with an increased risk of ovarian cancer. However, it has since been shown that infertility itself is a risk factor for ovarian cancer, and the risk observed may only be a reflection of the infertility status of these patients. Nevertheless, it would be prudent to avoid the unnecessary use of fertility drugs, and the old practice of empirically giving infertility patients months or even years of 'fertility pills' to augment their fertility should be abandoned.

Limitations of ART are not only related to the medical consequences. In most countries treatment is not funded or is only partially funded by the government. For the most part, couples have to fund their own treatment, and the costs involved are prohibitive to many. In addition, there are ethical considerations for many couples, whether they be religious or otherwise, that create a barrier to treatment.

The psychological and emotional stress of assisted reproduction should not be underestimated. A significant emotional, financial and temporal commitment is required for a couple to undertake a cycle of treatment. This places their relationship under even more stress than may have existed before commencing treatment. Trained fertility counsellors are an integral part of a treatment program and couples should be encouraged to make use of them.

\section{CONCLUSIONS}

The development of IVF and its associated technologies has not only revolutionized the treatment of infertility, but has also created a worldwide industry dedicated to its success. It has become so successful that it is now a relatively rare occurrence when no treatment alternatives are available to an individual couple. Continued research in this area is likely to lead to higher success rates in the future.

Along with the progress of ART, there have also been associated complications in the form of iatrogenic OHSS and a significant increase in the number of higher order multiple pregnancies. Newer techniques such as IVM and aneuploidy screening may help reduce some of these associated risks and improve outcomes even further.

Access to treatment may be limited by social and economic constraints, and, in addition, there have been and will continue to be ethical controversies surrounding some of the available treatments. The speed of development of assisted reproduction has meant that society has, in many cases, not been able to adjust to these ethical situations or develop adequate guidelines.

The success of ART will no doubt continue to improve with higher pregnancy rates, reduced risks and complications, and more affordable and patient friendly treatments.

\section{REFERENCES}

1. Steptoe PC, Edwards RG. Birth after re-implantation of a human embryo. Lancet 1978;2:366. (Lett)

2. Tan SL, Steer C, Royston P, et al. Conception rates and in-vitro fertilisation. Lancet 1990;335:299.

3. Tan SL, Royston P, Campbell S, et al. Cumulative conception and livebirth rates after in-vitro fertilisation. Lancet 1992;339:1390-4.

4. Engmann L, Maconochie N, Bekir JS, et al. Cumulative probability of clinical pregnancy and live birth after a multiple cycle IVF package: A more realistic assessment of overall and age-specific success rates? Br J Obstet Gynaecol 1999;106:165-70.

5. MacDougall MJ, Tan SL, Balen A, Jacobs HS. A controlled study comparing patients with and without polycystic ovaries undergoing in-vitro fertilization. Hum Reprod 1993;8:233-7.

6. Zaidi J, Barber J, Kyei-mensah A, et al. Relationship of ovarian stromal blood flow at the baseline ultrasound scan to subsequent follicular response in an in vitro fertilization program. Obstet Gynecol 1996;88:779-84. 
7. Engmann L, Sladkevicius P, Agrawal R, Bekir JS, Campbell S, Tan SL. Value of ovarian stromal blood flow velocity measurement after pituitary suppression in the prediction of ovarian responsiveness and outcome of in vitro fertilisation treatment. Fertil Steril 1999;71:22-9.

8. Tan SL, Maconochie N, Doyle P, et al. Cumulative conception and live-birth rates after in vitro fertilization with and without the use of long, short, and ultrashort regimens of the gonadotropin-releasing hormone agonist buserelin. Am J Obstet Gynecol 1994;171:513-20.

9. Balen AH, Tan SL, MacDougall J, Jacobs HS. Miscarriage rates following in-vitro fertilization are increased in women with polycystic ovaries and reduced by pituitary desensitization with buserelin. Hum Reprod 1993;8:959-64.

10. Tan SL, Kingsland C, Campbell S, et al. The long protocol of administration of gonadotropin-releasing hormone agonist is superior to the short protocol for ovarian stimulation for in vitro fertilization. Fertil Steril 1992;57:810-4.

11. Shoham Z. The clinical therapeutic window for luteinizing hormone in controlled ovarian stimulation. Fertil Steril 2002;77:1170-7.

12. Tan SL, Bennett S, Parsons J. Surgical techniques of oocyte collection and embryo transfer. Br Med Bull 1990;46:628-42.

13. Tan SL, Waterstone J, Wren M, Parsons J. A prospective randomized study comparing aspiration only with aspiration and flushing for transvaginal ultrasound-directed oocyte recovery. Fertil Steril 1992;58:356-60

14. Senn A, Vozzi C, Chanson A, et al. Prospective randomized study of two cryopreservation policies avoiding embryo selection: The pronucleate stage leads to a higher cumulative delivery rate than the early cleavage stage. Fertil Steril 2000;74:946-52.

15. Gosden RG, Baird DT, Wade JC, et al. Restoration of fertility to oophorectomized sheep by ovarian autografts stored at $-196^{\circ} \mathrm{C}$. Hum Reprod 1994;9:597-603.

16. Oktay K, Karlikaya G. Ovarian function after transplantation of frozen, banked autologous ovarian tissue. N Engl J Med 2000;342:1919.

17. Kuleshova L, Gianaroli L, Magli C, et al. Birth following vitrification of a small number of human oocytes: case report. Hum Reprod 1999; 14:3077-9.

18. Trounson A, Leeton J, Besanko M, et al. Pregnancy established in an infertile patient after transfer of a donated embryo fertilised in vitro. Br Med J 1983;286:835-8.

19. Cha K, Koo J, Ko J, et al. Pregnancy after in vitro fertilization of human follicular oocytes collected from nonstimulated cycles, their culture in vitro and their transfer in a donor oocyte program. Fertil Steril 1991;55:109-13.

20. Chian R, Buckett W, Tulandi T, et al. Prospective randomized study of human chorionic gonadotrophin priming before immature oocyte retrieval from unstimulated women with polycystic ovarian syndrome. Hum Reprod 2000;15:165-70.
21. Tan SL, Child TJ. In-vitro maturation of oocytes from unstimulated polycystic ovaries. Reprod Biomed Online 2001;4(Suppl 1):18-23.

22. Whittemore AS. The risk of ovarian cancer after treatment for infertility. N Engl J Med 1994;331:805-6.

23. Tan SL, Child TJ, Gulekli B. In vitro maturation and fertilization of oocytes from unstimulated ovaries: Predicting the number of immature oocytes retrieved by early follicular phase ultrasonography. Am J Obstet Gynecol 2002;186:684-9.

24. Child TJ, Abdul-Jalil AK, Gulekli B, Tan SL. In vitro maturation and fertilization of oocytes from unstimulated normal ovaries, polycystic ovaries, and women with polycystic ovary syndrome. Fertil Steril 2001;76:936-42.

25. Chian R, Gulecki B, Buckett W, et al. Priming with human chorionic gonadotrophin before retrieval of immature oocytes in women with infertility due to polycystic ovarian syndrome. N Engl J Med 1999;341:1624-6.

26. Handyside A, Kontogianni E, Hardy K, et al. Pregnancies from biopsied human preimplantation embryos sexed by Y-specific DNA amplification. Nature 1990;344:768-70.

27. Munne S, Wells D. Preimplantation genetic diagnosis. Curr Opin Obstet Gynecol 2002;14:239-44.

28. Munne S, Cohen J, Sable D. Preimplantation genetic diagnosis for advanced maternal age and other indications. Fertil Steril 2002;78:234-6.

29. Wells D, Delhanty JD. Comprehensive chromosomal analysis of human preimplantation embryos using whole genome amplification and single cell comparative genomic hybridization. Mol Hum Reprod 2000;6:1055-62.

30. Engmann L, Maconochie N, Bekir J, et al. Cumulative probability of clinical pregnancy and live birth after a multiple cycle IVF package: A more realistic assessment of overall and age specific success rates? Br J Obstst Gynaecol 1999;106:165-70.

31. Stewart JA, Hamilton PJ, Murdoch AP. Thromboembolic disease associated with ovarian stimulation and assisted conception techniques. Hum Reprod 1997;12:2167-73.

32. Jain T, Harlow BL, Hornstein MD. Insurance coverage and outcomes of in vitro fertilisation. N Engl J Med 2002;347:661-6.

33. 1999 Assisted Reproductive Technology Success Rates. $<$ http://www.cdc.gov/nccdphp/drh/art99/sample.htm> Version current at October 2002.

34. Tan SL, Doyle P, Campbell S, et al. Obstetric outcome of in vitro fertilization pregnancies compared with normally conceived pregnancies. Am J Obstet Gynecol 1992;167:778-84.

35. Saunders DM, Lancaster P. The wider perinatal significance of the Australian in vitro fertilization data collection program. Am J Perinatol 1989;6:252-7.

36. Bonduelle M, Liebaers I, Deketelaere V, et al. Neonatal data on a cohort of 2889 infants born after ICSI (1991-1999) and of 2995 infants born after IVF (1983-1999). Hum Reprod 2002;17:671-94. 